\title{
Dominator Color class Dominating sets on Ladder, Open Ladder and Slanting Ladder Graphs
}

Niju $\mathrm{P}^{1}$ and Vijayalekshmi $\mathrm{A}^{2}$

Received: 21 October 2021/ Accepted: 10 December 2021/ Published online: 21 December 2021

CSacred Heart Research Publications 2017

\begin{abstract}
A proper coloring $\mathcal{C}=\left\{\mathcal{C}_{1}, \mathcal{C}_{2}, \ldots \ldots \ldots \mathcal{C}_{\chi}\right\}$ of a graph $G=(V, E)$ is a dominator color class dominating set of $G$ if each $v \in V(G)$ is dominated by a color class $\mathcal{C}_{i} \in$ and each $\mathcal{C}_{i} \in \mathcal{C}$ dominates a vertex $u \in V(G)$. The minimum number of color classes that satisfy the above condition is known as dominator color class domination number of $G$ and is denoted by $\gamma_{\chi}^{d}(G)$. In this paper, we find the dominator color class domination number of ladder, open ladder and slanting ladder graphs.
\end{abstract}

Key Words: dominator color class dominating sets, dominated color class domination number.

AMS Classification: 05C69.

\section{Introduction}

In this paper, we only consider ladder,open ladder and slanting ladder graphs. Further terminology can be found in [3].

A proper coloring $\mathcal{C}=\left\{\mathcal{C}_{1}, \mathcal{C}_{2}, \ldots \ldots \ldots \mathcal{C}_{\chi}\right\}$ of a graph $G=(V, E)$ is a dominator color class dominating set of $G$ if each $v \in V(G)$ is dominated by a color class $\mathcal{C}_{i} \in$ and each $\mathcal{C}_{i} \in \mathcal{C}$ dominates a vertex $u \in V(G)$. The minimum number of color classes that satisfy the above condition is known as dominator color class domination number of $G$ and is denoted by $\gamma_{\chi}^{d}(G)$.This notion was introduced by A. Vijayalekshmi and P. Niju in [2].

\footnotetext{
${ }^{1}$ Department of Mathematics, S.T. Hindu College, Nagercoil-629002, Tamilnadu, India.

${ }^{2}$ Department of Mathematics, S.T. Hindu College, Nagercoil-629002, Tamilnadu, India. Email: vijimath.a@gmail.com
} 
The cartesian product of two graphs $G_{1}=\left(V_{1}, E_{1}\right)$ and $G_{2}=\left(V_{2}, E_{2}\right)$, denoted by $G_{1} \times G_{2}$ is a graph with vertex set $V_{1} \times V_{2}$ and edges between two vertices $\left(u_{1}, v_{1}\right)$ and $\left(u_{2}, v_{2}\right)$ if and only if either $u_{1}=u_{2}$ and $v_{1} v_{2} \in E\left(G_{2}\right)$ (or) $u_{1} u_{2} \in E\left(G_{1}\right)$ and $v_{1}=v_{2}$. A ladder graph can be defined as $P_{2} \times P_{n}$ where $n \geq 2$ and is denoted by and $L_{p}\left|V\left(L_{p}\right)\right|=2 n, n \geq 2$. An open ladder graph $O\left(L_{p}\right)$, is obtained from two paths of length $n-1$ with $V\left(L_{p}\right)=\left\{u_{i} v_{i} / 1 \leq i \leq\right.$ $n\}$ and $E\left(L_{p}\right)=\left\{u_{i} u_{i+1}, v_{i} v_{i+1} / 1 \leq i \leq n-1\right\} \cup\left\{u_{i} v_{i}: 2 \leq i \leq n-1\right\}$. A slanting ladder graph $S L_{p}=S L_{2 n}(n \geq 3)$ is agraph obtained from two paths $u_{1}, u_{2} \ldots \ldots u_{n}$ and $v_{1}, v_{2} \ldots \ldots v_{n}$ by joining each $u_{i}$ with $v_{i+1}(1 \leq i \leq n-1)$. In this paper, we obtain dominator color class domination number of ladder graph, open ladder and slanting ladder graph.

\section{Main Results}

Theorem 2.1. Let $L_{p}$ be a ladder graph of order $2 n$. Then $\gamma_{\chi}^{d}\left(L_{p}\right)=\left\{\begin{array}{ll}p & \text { if } p \text { is even } \\ p+1 & \text { if } p \text { is odd }\end{array}\right.$.

Proof: Let $L_{p}=L_{2 n}=p_{2} \times p_{n}$ and let $V\left(L_{p}\right)=\left\{u_{1}, u_{2}, \ldots \ldots . u_{2 n}\right\}$ withdeg $u_{i}=2$ for $i=1, n,(n+1), 2 n$ and $\operatorname{deg} u_{j}=3$ for all $j \neq i$.

We take $N\left(u_{i}\right)=\left\{u_{i-1}, u_{i+1}, u_{i+n}\right\}$ for $i=2,3, \ldots \ldots \ldots(n-1)$ and $N\left(u_{j}\right)=\left\{u_{j-1}, u_{j+1}, u_{j-n}\right\}$ for $j=(n+2),(n+3), \ldots \ldots(2 n-1)$. We consider two cases

Case (1): When $p \equiv 0(\bmod 2)$

Decompose $L_{p}$ in to $\frac{p}{2}$ copies of $L_{2}$. Assign new colors, say $2 i-1,2 i\left(1 \leq i \leq \frac{p}{2}\right)$ to the vertices $\left\{u_{2 i-1}, u_{2 i+n}\right\}$ and $\left\{u_{2 i}, u_{2 i+(n-1)}\right\}$ respectively. We attain a $\gamma_{\chi}^{d}$ coloring of $L_{p}$. So $\gamma_{\chi}^{d}\left(L_{p}\right)=p$.

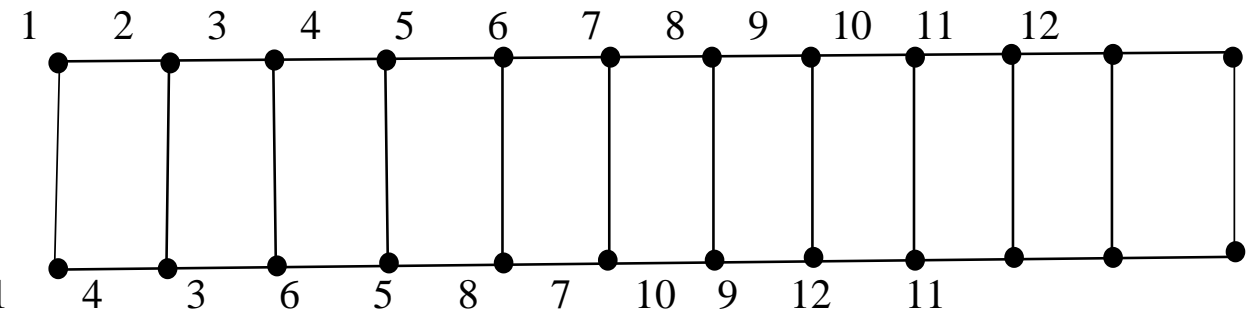

Figure1 $\gamma_{\chi}^{d}\left(L_{12}\right)=12$ 
Case (2): When $p \equiv 1(\bmod 2)$

Since $p-1 \equiv 0(\bmod 2), L_{p}$ is obtained from, $L_{p-1}$ followed by $p_{2}$. As in case (i) $\gamma_{\chi}^{d}\left(L_{p-1}\right)=p-1$. Assign two new colors, say, $p$ and $p+1$ to the vertices $\left\{u_{n}\right\}$ and $\left\{u_{2 n}\right\}$ respectively. We get a $\gamma_{\chi}^{d}$ - coloring of $L_{p}$. Thus

$$
\gamma_{\chi}^{d}\left(L_{p}\right)=p+1
$$

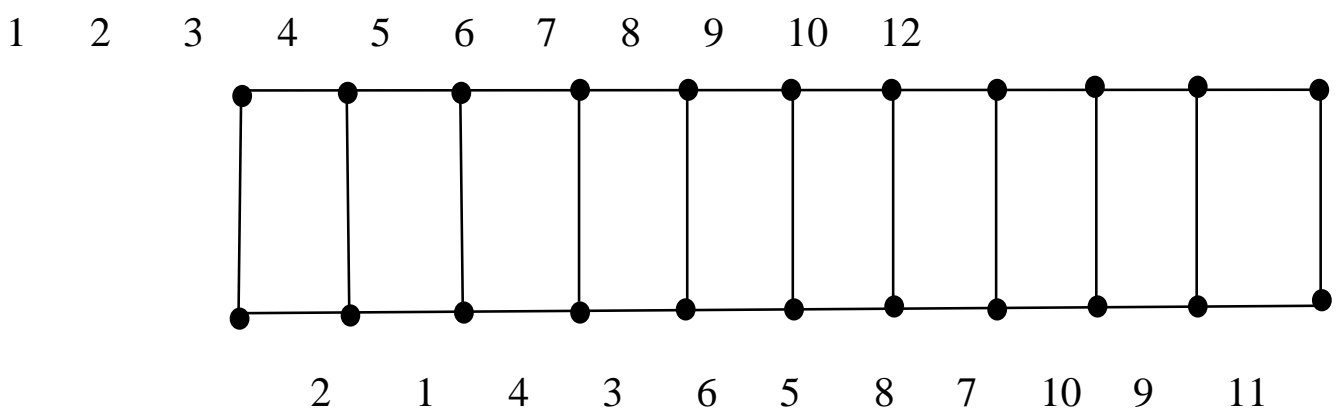

Figure2 $\gamma_{\chi}^{d}\left(L_{11}\right)=12$

Theorem 2.2. Let $O\left(L_{p}\right)$ be an open ladder graph. Then

$$
\gamma_{\chi}^{d}\left(O\left(L_{p}\right)=\left\{\begin{array}{c}
p+1 \text { if } p \equiv 1(\bmod 4) \\
p+2 \quad \text { otherwise }
\end{array}\right.\right.
$$

Proof: Let $V\left(O\left(L_{p}\right)\right)=\left\{u_{1}, u_{2}, \ldots \ldots \ldots \ldots \ldots u_{2 n}\right\}$ with $\operatorname{deg} u_{i}=1$

for $i=1, n,(n+1), 2 n$ and $\operatorname{deg} u_{j}=3 \forall j \neq i$.

We have 2 cases.

Case (1): When $p \equiv 1(\bmod 4)$

Assign four distinct colors, say 1,2, $p$ and $p+1$ to the pendent vertices $\left\{u_{1}\right\},\left\{u_{n}\right\},\left\{u_{n+1}\right\}$ and $\left\{u_{2 n}\right\}$ respectively.

Let $H=\left\langle u_{2}, \ldots . . u_{n-1}, u_{n+1}, \ldots \ldots u_{2 n-1}\right\rangle$. Then $H \cong L_{p-2}$. Decompose $H$ into $\left\lfloor\frac{P-2}{4}\right\rfloor$ copies of $L_{4}$ and one copy of $L_{3}$. 
Assign distinct colors $4 i-1,4 i, 4 i+1$ and $4 i+2\left(1 \leq i \leq\left\lfloor\frac{p-2}{4}\right\rfloor\right.$ to the vertices $\left\{u_{4 i-1}, u_{n+4 i-2}, u_{n+4 i}\right\},\left\{u_{4 i-2}, u_{4 i}, u_{n+4 i-1}\right\},\left\{u_{4 i+1}\right\}$ and $\left\{u_{n+4 i+1}\right\}$

respectively. Also assign 2 distinct colors say, $(p-2)$ and $(p-1)$ to the vertices $\left\{u_{n-3}, u_{n-1}, u_{2 n-2}\right\}$ and $\left\{u_{n-2}, u_{2 n-3}, u_{2 n-1}\right\}$ respectively, we attain a $\gamma_{\chi}^{d}-$ coloring of $O\left(L_{p}\right)$. Thus $\gamma_{\chi}^{d}\left(O\left(L_{p}\right)\right)=p+1$.

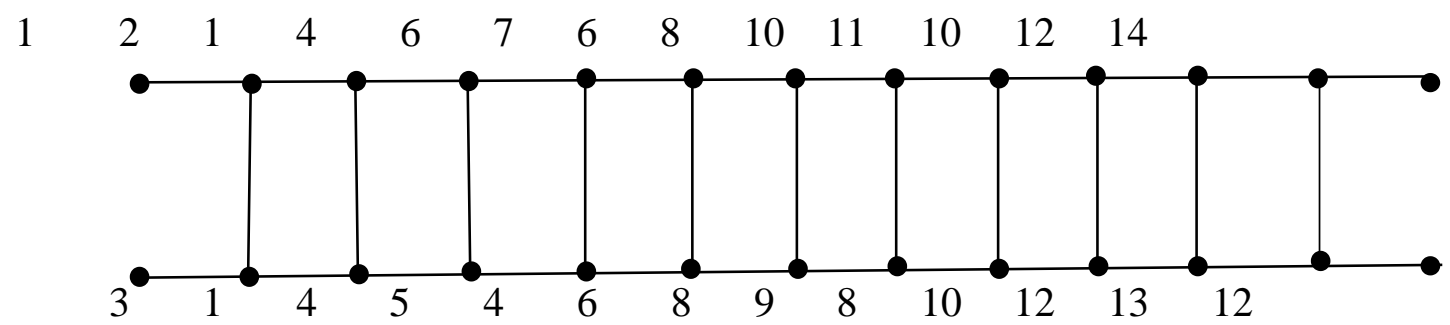

Figure $3 \quad \gamma_{\chi}^{d}\left(O\left(L_{13}\right)\right)=14$

Case (2): When $p \equiv 0,2,3(\bmod 4)$

We have three subcases

Subcase 2.1: When $p \equiv 0(\bmod 4)$

Assign 4 distinct colors say $1,2,(p+1)$ and $(p+2)$ to the pendant vertices of $L_{p}$. As in case (1) $H$ is decomposed into $\left\lfloor\frac{p-2}{4}\right\rfloor$ copies of $L_{4}$ and one copy of $L_{2}$.Assign distinct colors, say $4 i-1,4 i, 4 i+1$ and $4 i+2\left(1 \leq i \leq\left\lfloor\frac{p-2}{4}\right\rfloor\right.$ to the same vertices as in case (1). Also assign 2 distinct colors say $(p-1)$ and $p$ to the vertices $\left\{u_{n-2}, u_{2 n-1}\right\}$ and $\left\{u_{n-1}, u_{2 n-2}\right\}$ respectively. We attain a $\gamma_{\chi}^{d}$ - coloring of $L_{p}$. Thus $\gamma_{\chi}^{d}\left(O\left(L_{p}\right)\right)=p+2$.

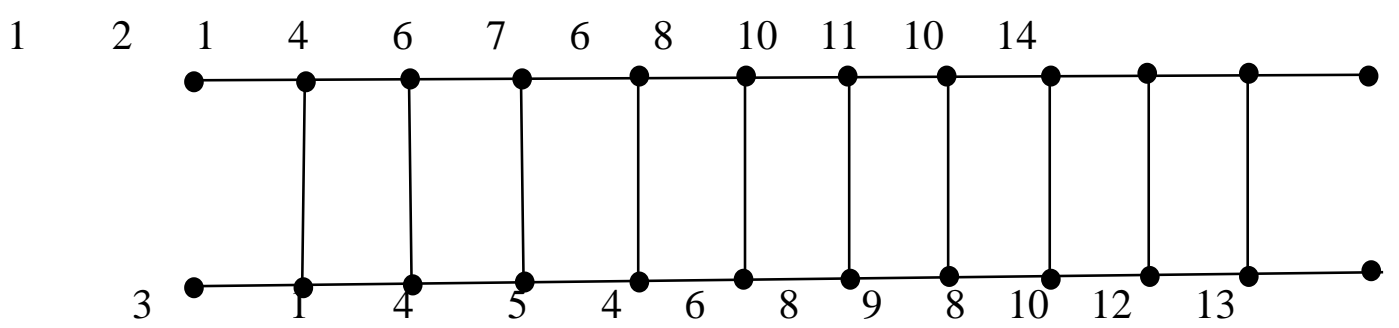

Figure4 $\quad \gamma_{\chi}^{d}\left(O\left(L_{12}\right)\right)=14$ 
Subcase 2.2: When $p \equiv 2(\bmod 4)$

Since $p-2 \equiv 0(\bmod 4)$ and as in case (i), $H$ can be decomposed into $\frac{p-2}{4}$ copies of $L_{4}$. Assign each 4 distinct colors $4 i-1,4 i, 4 i+1$ and $4 i+2$ $\left(1 \leq i \leq \frac{p-2}{4}\right)$ to the said vertices above, we get a $\gamma_{\chi}^{d}-$ coloring of $L_{p}$. So $\gamma_{\chi}^{d}\left(L_{p}\right)=4+$ $\left(\frac{p-2}{4}\right) 4=p+2$.

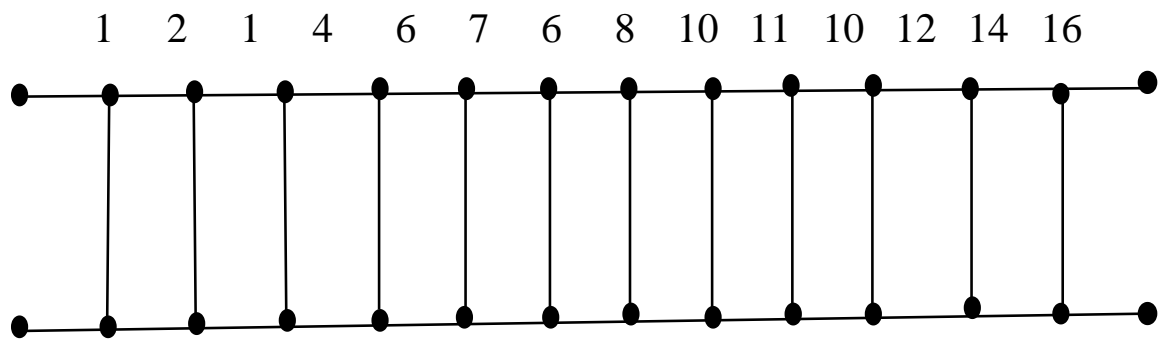

$\begin{array}{llllllllllllll}3 & 1 & 4 & 5 & 4 & 6 & 8 & 9 & 8 & 10 & 12 & 13 & 12 & 15\end{array}$

Figure $5 \quad \gamma_{\chi}^{d}\left(L_{14}\right)=16$

Subcase 2.3: When $p \equiv 3(\bmod 4)$

Assign 3 distinct colors say 1,2 and $(p+2)$ to the pendant vertices say $\left\{u_{1}\right\},\left\{u_{2}\right\}$

and $\left\{u_{2 n}\right\}$ respectively. Let $H=\left\langle u_{2}, \ldots . u_{n-2}, u_{n+2}, u_{n+3}, \ldots . u_{2 n-2}\right\rangle$ and $H$ can be decomposed in $\left\lfloor\frac{p-2}{4}\right\rfloor$ copies of $L_{4}$. Apply the same coloring to thevertices in $H$. Also apply two distinct colors say, $p$ and $p+1$ to the vertices $\left\{u_{n-1}\right\}$ and $\left\{u_{n}, u_{2 n-1}\right\}$ respectively, we obtain a $\gamma_{\chi}^{d}$ - coloring of $L_{p}$. So $\gamma_{\chi}^{d}\left(L_{p}\right)=p+2$.

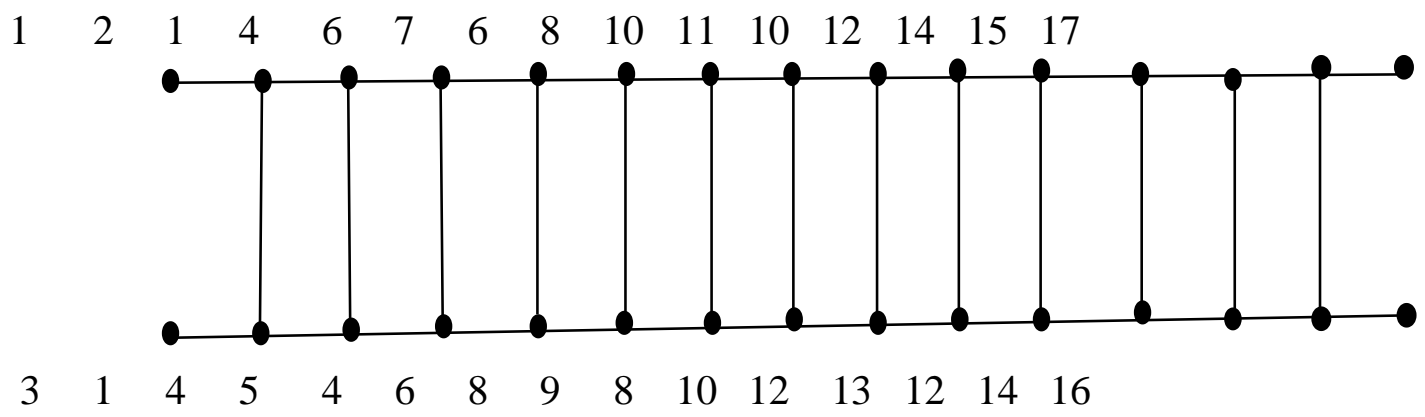

Figure $6 \quad \gamma_{\chi}^{d}\left(L_{15}\right)=17$ 
Theorem 2.3. Let $S L_{n}$ be a slanting ladder graph. Then $\gamma_{\chi}^{d}\left(S L_{n}\right)=n+1 \forall n \geq 3$

Proof: Let $S L_{p}=S L_{2 n}(n \geq 3)$ be a slanting ladder graph with $V\left(S L_{2 n}\right)=$ $\left\{u_{1}, u_{2}, \ldots \ldots . u_{n}, v_{1}, v_{2}, \ldots \ldots v_{n}\right\}$ and $E\left(S L_{2 n}\right)=\left\{u_{i} u_{i+1} \mid i<n\right\} \cup\left\{v_{i} v_{i+1} \mid i<n\right\} \cup$ $\left\{u_{i} v_{i+1} \mid 1 \leq i \leq n-1\right\}$.

Assign 3 distinct colors say $1, n-1$ and $n$ to the vertices say $\left\{v_{1}\right\},\left\{v_{n}\right\}$ and $\left\{u_{n}\right\}$ respectively

Assign distinct colors say $3 i+1\left(1 \leq i \leq\left\lfloor\frac{n}{3}\right\rfloor\right)$ to the vertices $\left\{v_{3 i+1}\right\} 1 \leq i \leq\left\lfloor\frac{n}{3}\right\rfloor$. Also assign distinct colors say $3 j-1$ and $3 j\left(1 \leq j \leq\left\lfloor\frac{n}{3}\right\rfloor\right)$ to the vertices $\left\{u_{3 i-2}, u_{3 i}, v_{3 i}\right\}$ and $\left\{u_{3 i-1}, v_{3 i-1}\right\}$ respectively, we obtain a $\gamma_{\chi}^{d}$ - coloring of $S L_{n}$. Thus $\gamma_{\chi}^{d}\left(S L_{n}\right)=n+1 \forall n \geq$ 3.

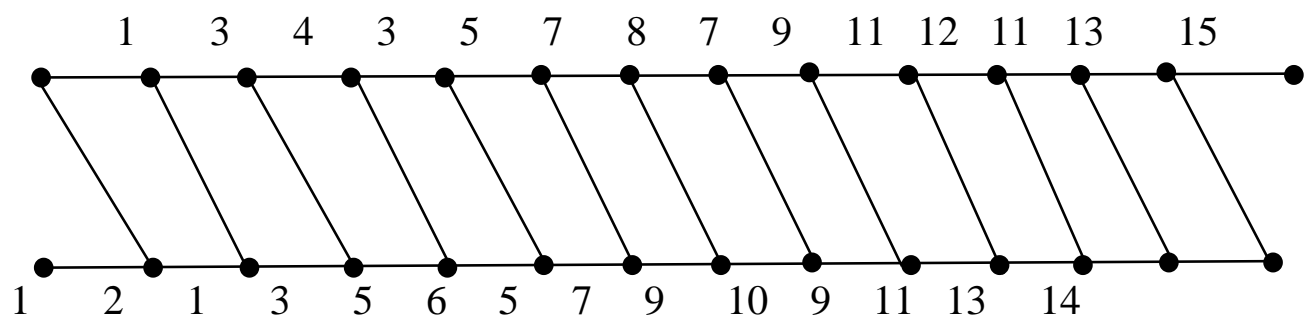

Figure7 $\gamma_{\chi}^{d}\left(S L_{14}\right)=15$

\section{References}

[1] A. Vijayalekshmi, A.E Prabha, Introduction of color class dominating sets in Graphs, Malaya Journal of Matematik, Vol.8, No.4,2186-2189,2020.

[2] A. Vijayalekshmi, P. Niju, An Introduction of Dominator color class dominating sets in Graphs, Malaya Journal of Matematik, Vol.9, No.1, 2021.

[3] F. Harrary, Graph theory, Addition -Wesley Reading Mass, 1969

[4] TerasaW. Haynes, Stephen T. Hedetniemi, Peter Slater, Domination in graphs, Marcel Dekker, New York, 1998. 